\title{
LE JOURNAL PERSONNEL EN TANT QUE DISPOSITIF CRITIQUE ET DIDACTIQUE
}

\begin{abstract}
A bstract. Lis Jerzy, Le journal personnel en rant que dispositif critique et didactique [Personal diary as a critical and didactic instrument]. Studia Romanica Posnaniensia, Adam Mickiewicz University Press, Poznań, vol. XXVIII: 2001, pp. 117-130, ISBN 83-232-1114-2, ISSN 0137-2475.

An intimate diary is not only and exclusively spontaneous form of writing on personal themes, but may also appear as an instrument of critical reflection, and as a didactic aid. On the example of scientfic works of Philippe Lejeune and a handbook for pupils who have learning difficulties, the author analyses directions of development of this form of autobiographical recording and possibilities of using a diary in domains other than writing literature.
\end{abstract}

La pratique du journal personnel est aujourd'hui une réalité scrupturaire confirmée dont on ne saurait surestimer la portée ni du point de vue quantitatif ni qualitatif. Naturelle à bien des égards pour des professionnels de l'écriture, la tenue du journal est avant tout un droit intime et social des hommes ordinaires à l'expression par écrit. L'histoire du journal personnel est là pour prouver que la pratique telle quelle n'est pas réservée aux élus et qu'elle s'inscrit dans le goût de l'individu pour une autoanalyse. Compte tenu du fait qu'aussi bien la réception des journaux que leur situation générique constituent depuis quelque temps l'objet des études systématiques approfondies, mes réflexions seront centrées sur deux modes particuliers de la pratique du journal, les deux visant d'autres objectifs et ayant pourtant pour principe de base les mêmes tics, avantages, facilités et contraintes de l'écriture autobiographique.

J'ai choisi pour cette présentation deux cas, à mon avis très instructifs pour ce qui est de leurs horizons d'attente respectifs. L'originalité du dispositif critique en forme de journal consiste à mettre en pratique un discours à caractère scientifique à partir de notes recueillies selon les modalités du journal et dont la discursivité spontanée est à l'opposé de ce qu'on attend des textes de recherches rédigés comme tels. Je vais donc revisiter plusieurs travaux de Philippe Lejeune, spécialiste en autobiographie, chercheur et diariste à la fois qui se sert du journal dans ses travaux sur le 
genre même. Le second cas est envisagé comme dispositif didactique à l'usage des élèves en difficulté. Son essence consiste à mobiliser des adolescents à la pratique du journal intime en tant que mode d'entraînement dans l'expression libre suivant le modèle concret d'un journal personnel publié. Cette méthode d'enseignement rappelle en gros des ateliers d'écriture proposés aux amateurs de l'autobiographie ainsi que des manuels d'écriture autobiographique, très recherchés par les postulants à la gloire littéraire.

\section{JOURNAL DE RECHERCHE DE LEJEUNE}

Quand on feuillette la liste des publications de Ph. Lejeune sur l'écriture autobiographique, on se rend compte immédiatement de l'ampleur de son oeuvre critique et en même temps de la discipline thématique de ses recherches. Depuis la parution en 1971 de l'étude d'ensemble intitulée l'Autobiographie en France, Lejeune a publié une quinzaine de livres, tous ayant pour centre d'intérêt différents aspects de l'expression de soi. Le corpus est donc important, et si l'on y ajoute encore actes de colloques, bibliographies des études en langue française sur la littérature personnelle et les récits de vie, articles d'encyclopédie, préfaces, entretiens, articles publiés dans diverses revues, on obtient une somme impressionnante qui fait de ce chercheur une figure de proue de la critique autobiographique en France. Les recherches initiées par Jui sont fort originales, car elles vont un peu à l'encontre des méthodologies élaborées dans les années soixante-dix. Une autre originalité de Lejeune réside dans le fait qu'en dépit de plusieurs tournants qu'il a subis dans sa vie professionnelle, il est resté fidèle à ses démarches interprétatives des textes autobiographiques.

Dans tous les travaux de Lejeune nous trouverons des traces de la tension entre le vécu et la manière d'en parler, et surtout des traces de l'élaboration du discours critique capable d'exprimer l'essence de l'écriture autobiographique, et qui serait en mesure de décrire une réalité scrupturaire que les institutions universitaires passent sous silence. Quand il a commencé, en 1969, à travailler sur l'autobiographie, il s'est trouvé en présence du vide de la littérature critique sur le sujet et par conséquent toute son histoire personnelle du dernier quart du siècle s'est développée dans le cadre de la disparition successive du mépris vis-à-vis de ce genre méconnu (PA, 12)!

Comme c'est le cas de beaucoup d'individus, l'activité professionnelle est souvent le résultat des coïncidences et du concours de circonstances. Philippe Lejeune ne serait pas devenu chercheur s'il n'avait pas eu dans les années cinquante un coup de passion pour l'oeuvre de Rousseau et de Proust, les deux écrivains qui lui proposaient, il est vrai - sans aucune arrière-pensée autobiographique, des moyens de construction de l'existence individuelle, c'est-à-dire des moyens de voir comment la vie d'un in-

${ }^{1}$ La liste bibliographique de tous les textes mentionnés (et de leurs abréviations dont je me sers) est jointe à la fin de cette communication. 
dividu pouvait, en s'écrivant, obtenir forme et sens. Cette découverte n'était sans importance ni pour les décisions concernant sa carrière d'enseignant universitaire, ni pour les projets de recherche qu'il devait mettre bientôt en oeuvre. Entre 1969 et 1986, la plupart de ses publications étaient consacrées à l'autobiographie contre le journal. Le début du travail universitaire nécessitait une espèce de maîtrise de soi liée avec le besoin de reconstruction de sa vie.

Après la découverte de Proust et de Rousseau, cela aura été pour lui le deuxième tournant important, lequel, en tant que moment critique de l'existence individuelle et à la fois forme brève de l'autobiographie, consistait à envisager l'illustration par écrit d'une interrogation banale: comment je suis devenu moi (cf. TT, 8-9). Lejeune ne cache pas que depuis 1969 sa vie a changé, car il a découvert qu'il existait un art de l'autobiographie (S, 238) et qu'il valait la peine de se lancer dans l'étude de ce genre. En effet, il aura publié sept livres dont L'Autobiographie en France (1971, A. Collin), Exercices d'ambiguittés. Lectures de "Si le grain ne meurt» (1974, Lettres Modernes), Lire Leiris. Autobiographie et langage (1975, Klincksieck), Le Pacte autobiographique (1975, Seuil), Je est un autre. L'Autobiographie de la littérature aux médias (1980, Seuil), Moi aussi (1986, Seuil). A cette liste il faut absolument ajouter la publication de l'autobiographie de son arrière-grand-père Xavier-Edouard Lejeune ( $\mathrm{Ca}$ licot. Enquête de Michel et Philippe Lejeune, 1984, éd. Montalba). Cette oeuvre critique est aujourd'hui généralement connue.

A la même époque, en dehors de ses travaux scientifiques, Lejeune cherchait également une forme convenable pour la description de soi-même et a essayé de composer des textes autobiographiques à la Leiris (PA, 222). Une tentative qui a échoué, mais qui lui a permis d'entrevoir une autre possibilité. Il venait juste de reprendre son journal abandonné depuis quelques années et a compris que le montage des fragments de journaux intimes pouvait bien se présenter comme un acte autobiographique. La lecture du Temps immobile de Claude Mauriac, publié en volumes entre 1974 et 1988, a été décisive (TJ, 360), bien que le journal fût lié à l'idée de détresse et de dérive.

L'intérêt de Lejeune pour le journal intime en tant que genre peut être justifié par la situation paradoxale des études autobiographiques en France. Excepté quelques travaux de Gusdorf et de Starobinski, il n'y avait à l'époque aucune étude d'ensemble sur l'autobiographie (Lejeune comblera cette lacune dans les années soixante-dix), alors que plusieurs monographies sur le journal intime (M. Leleu, A. Girard, B. Didier) étaient déjà connues des amateurs. En tant que diariste, Philippe Lejeune était conscient que l'étude des textes publiés posait de grands problèmes de l'échantillonnage (publier un texte d'écrivain ou celui de personne ordinaire) et de la transformation (publier intégralement ou en fragments), et qu'il était nécessaire de considérer le journal comme une pratique plutôt qu'un texte. C'est pourquoi entre 1987 et 1997 , Lejeune s'est entièrement consacré à l'étude de la pratique diaristique qui, avant de comprendre la dimension historique et théorique du problème, lui a permis d'avancer une hypothèse suivante: «la pratique du journal est liée à la scolarisation des adoles- 
cents. L'école obligatoire pour tous et la prolongation des études n'ont pu que la développer» (TJ, 363).

Les résultats de ses investigations ont été publiés successivement dans les monographies suivantes: La Pratique du journal personnel. Enquête (1990, Publidix, Université Paris-X), «Cher cahier...» Témoignages sur le journal personnel recueillis et présentés par Philippe Lejeune (1990, Gallimard), Le journal personnel (1993, Publidix, Université Paris-X - colloque de mai 1990, sous la dir. de Ph. Lejeune), Archives autobiographiques (1991, dir. Ph. Lejeune, «Cahiers de sémiotique textuelle»), Le Moi des demoiselles. Enquête sur le joumal de jeune fille (1993, Seuil), Lucile Desmoulins. Journal 1788-1793 (1995, Ed. des Cendres, texte établi et présenté par Ph. Lejeune), Pour l'autobiographie (1998, Seuil), Les Brouillons de soi (1998, Seuil), „Cher écran...” (2000, Seuil). De nombreux articles parus dans diverses revues complètent cette liste et témoignent de l'ampleur de ses recherches sur la partique du journal personnel.

A la lumière de ces textes on peut constater qu'à partir de 1987 Lejeune a dépassé le stade de l'hésitation permanente entre l'intime et le public. Une série d'expériences personnelles qui s'enchaînent naturellement dans un discours autobiographique d'homme et de chercheur est à l'origine de sa théorie du journal. Je me limiterai à la simple énumération de ces expériences: 1 . à l'âge de quinze ans, Lejeune a commencé son journal intime qu'il tenait pendant une dizaine d'années (MD, 122; TJ, 359); 2. durant des années, outre les journaux publiés d'écrivains et d'hommes célèbres, il ne connaissait que le sien (PA, 60); 3. il savait que son journal reste à l'état de manuscrit, mais que c'est un vrai journal (PA, 60); 4. il était persuadé que presque aucun journal n'a été publié comme il avait été écrit (B, 322); 5. au moment de la publication du Pacte autobiographique en 1975 il a décidé de passer de «nous» au «je» (PA, 24); 6. en 1986, il a découvert qu'il y a un art du journal; 7. à l'âge de cinquante ans il est revenu au journal avec des supports plus modernes: d'abord la machine à écrire et ensuite l'ordinateur (TJ, 360); 8. le journal qu'il tenait, et tient toujours, n'est pas un brouillon et il n'a pas de brouillon (B, 9); 9 . il a appris à écrire son journal comme un texte et à le structurer comme une autobiographie - toute publication anthume fait envisager une construction (AP, 61).

Le retour à la pratique du journal était donc un acte salutaire pour la théorie du journal, car tout en contestant le caractère médicale et moralisateur du discours critique des années soixante et soixante-dix (AP, 62), Lejeune a eu l'audace de proposer une autre forme de discours, un discours construit sur soi. Lors de la conférence sur l'écriture autobiographique qui s'est tenue à Cracovie en avril 1993 il a déclaré qu' «il est aventureux de mélanger les genres, en donnant forme autobiographique à un discours à visée scientifique sur l'autobiographie. On ne doit plus seulement convaincre, comme un savant, mais séduire» (WSP, 15). Il ne fait pas de doutes qu'il pensait alors au livre qu'il venait d'achever: Le moi des demoiselles, paru la même année. Le caractère scientifique du diarisme à l'usage de ses travaux critiques relève de la conviction que l'homme, dans la même mesure que le chercheur, doit accepter une espèce de pari sur l'avenir: «Le journal fonde la personne non pas dans la mêmeté, 
comme dirait Paul Ricoeur, puisqu'on aura changé, mais dans ce qu'il appelle l'ipséité, une sorte d'engagement abstrait de fidélité à soi» (PA, 225). Cette démarche a bien entendu ses avantages et ses inconvénients. Ferdinand Brunetière, grand résistant à l'autobiographie trouvait déjà que toutes les formes en rapport avec le moi étaient inacceptables dans la critique littéraire (PA, 15). En valorisant le «je», Ph. Lejeune a non seulement gagné en liberté d'expression, mais s'est assuré contre le langage neutre du discours scientifique.

Lors de la rédaction de «Cher cahier...» Témoignages sur le journal personnel, Lejeune partait du principe que l'écriture intime devrait demander une approche intime et qu'il était beaucoup plus intéressant d'explorer la pratique que d'analyser le contenu du journal. Plus il avançait dans ses enquêtes, plus il était persuadé que la notation diaire n'est pas essentiellement un privilège des grands écrivains. C'est une raison pour laquelle le parcours théorico-historique de Lejeune ne concerne que les journaux des hommes ordinaires. Il a commencé par la chose, semble-t-il, la plus facile, c'est-à-dire par les recherches sur la pratique d'aujourd'hui. «Cher cahier...» a donné le jour à une longue exploration du problème par l'intermédiaire de diverses activités: colloque sur le journal personnel, journées d'études sur les archives autobiographiques, la création de l'Association Pour l'Autobiographie, avant de passer à l'étude des journaux de jeunes filles au $X^{e}{ }^{e} s$, au $X X^{e} s$. et enfin au journal de jeune fille au XVIII ${ }^{\mathrm{e}} \mathrm{s}$. Les publications qui ont couronné les étapes successives de ses recherches ont été suivies en 1997 par une grande exposition «Un journal à soi», organisée à Lyon et conçue surtout comme une forme de publication des textes conservés dans les archives ou sauvegardés par les diaristes ou leurs proches.

Pour la période de ses recherches sur le diarisme, $\mathrm{Ph}$. Lejeune s'est servi de la forme de journal de recherche dans quatre publications: La pratique du journal personnel (éd. 1990), Le moi des demoiselles (éd. 1993), dans l'édition critique du Journal de Lucile Desmoulins (1995) et dans le texte publié dans le numéro d' «Autrement» consacré à la sincérité $(1995)^{2}$. L'étude de ces textes est en elle-même intéressante, car le lecteur a affaire à un discours qui ne correspond pas aux exigences des travaux universitaires et qui échappe aux règles de ce type d'exposés. La situation du lecteur est donc peu confortable, car il doit organiser sa lecture sur deux pistes: privée et publique. Dans le même texte ce sont deux hommes qui parlent: le chercheur et le diariste, même si dans le journal de recherche les informations d'ordre personnel nous sont livrées à petites doses. C'est la tension entre ces deux registres qui rend le texte passionnant. La dynamique de l'enquête scientifique s'oppose à la monotonie relative des entrées diaires, ce qui donne pour résultat un changement continu de rythme du texte que nous sommes en train de lire.

L'idée de tenir le journal de recherche lui est venue au moment où il voulait vérifier comment s'écrivaient des journaux de jeunes filles au XIX ${ }^{\mathrm{e}} \mathrm{s}$. Après avoir lancé

${ }^{2}$ Philippe Lejeune avoue avoir tenu encore un autre journal de recherche - «En marge de Cher cahier..., pendant deux ans, j'ai tenu un journal de recherche sans doute aussi intéressant que le livre lui-même, mais trop inextricablement mêlé à mon journal personnel pour être communiqué» (MD, 6I). 
un appel aux auditeurs de France-Culture sur l'état des archives familiales, Lejeune a reçu beaucoup de lettres et de journaux manuscrits, et faisait évidemment des notes de lectures qui sont vite devenues des notes diaristiques. Il a tenu ce journal de juillet 1991 à juillet 1992, et ce sont justement ces notes-ci qui font partie du corpus principal du livre. Suivent des lectures de textes choisis, le répertoire des journaux retrouvés lors de l'enquête et un autre journal d'enquête tenu en octobre/novembre 1992, joint en guise de bilan. Son enquête a permis d'inventorier des journaux qui datent dans la plupart des cas du Second Empire, mais on a droit de juger que cette pratique était déjà bien établie à l'époque dont témoignent quelques rares spécimens de la première moitié du $\mathrm{XIX}^{\mathrm{e}} \mathrm{s}$. ainsi qu'un journal de l'époque de la Révolution tenue par Camille Duplessis Desmoulins. Les notes de recherche consignées au rythme de ses analyses ont amené Lejeune à une typologie élémentaire, selon laquelle on distingue deux types de journaux - spirituel et laïque. L'échantillonnage auquel il procède ne laisse pas de doutes que le journal de jeune fille remplissait à l'époque une fonction éducative double - il était à la fois un examen de conscience et un exercice de rédaction. Dans ses notes à lui, Lejeune enregistre de courtes descriptions de journaux, fait des remarques concernant le style et la technique de la notation pour prouver la variété de registres et l'originalité de la démarche scripturale. En même temps il tient à démontrer le drame de la condition féminine au $\mathrm{XIX}^{\mathrm{e}} \mathrm{s}$. On voit que l'auteur est fasciné par ce monde disparu et qu'il a l'intention de rendre ces filles à la vie.

Dans le journal de recherche tenu en 1994 qui accompagne l'édition critique du Journal de Lucile Desmoulins, la méthode utilisée est la même, sauf que la méditation sur les enjeux est centrée davantage sur les coulisses du travail. Dans les deux textes mentionnés Lejeune se pose constamment des questions sur le problème de l'interprétation des textes analysés. Or, il est convaincu que toute information doit être séparée de la réflexion ( $\mathrm{L}, 121)$. Son travail est celui d'un archéologue plutôt que celui d'un biographe. Faire sortir de l'ombre les textes, essayer de dialoguer avec les diaristes sans leur imposer un point de vue de l'homme d'aujourd' hui. Lejeune répète à plusieurs reprises qu'il n'accepte pas de biographies où il s'agit de créer l'individu à partir d'interprétation des données factuelles - «Presque toutes les biographies sont des fictions [...] Le vrai se noie dans l'inventé et tout devient suspect. Le très peu qu'on connaît, on le dilue, on le pastiche, on le gonfle d'informations prises ailleurs, on le soutient de stéréotypes» (L, 117). Et pourtant, dans ses travaux l'interprétation côtoie le commentaire, mais ils sont séparés de façon à ce que l'objectif (un livre d'histoire) se distingue du subjectif (un livre de tendresse) (TJ, 371). Les textes qu'il analyse sont pris dans un sens important pour leurs auteurs eux-mêmes, il n'avance aucun jugement sur la qualité des carnets en privilégiant plutôt la voie de l'identification avec les scripteurs. Sur les pages du journal d'enquête on trouvera beaucoup de passages où apparaissent des bribes de réflexions, des fragments de synthèse, ou encore des essais de périodisation des journaux de jeunes filles.

Est-il donc possible de présenter les résultats de recherches scientifiques à l'aide d'une forme informe selon l'expression de Charles Du Bos? En effet, Lejeune trouve 
une solution un peu radicale, mais qui convient à la poétique du journal: transmettre au lecteur sa passion de journaux et raconter la genèse et l'évolution de sa propre enquête. Voilà ce qu'il dit à ce propos dans un article consacré à l'autogenèse: «Inutile de se retirer dans l'impersonnalité, en laissant son lecteur devant la masse redevenue inerte de manuscrits scientifiquement décrits, mais mornes et morts. Le mouvement de mon enquête, que je puis peindre, donnera une image analogue de ce qui est au fond l'objet perdu que nous cherchons à travers ces brouillons et ces ratures: le mouvement de la création. Il est plus intéressant de visiter un champ de fouilles avec l'archéologue que de voir des tessons alignés dans une vitrine. Et ce n'est pas moins scientifique. Les études génétiques sont faites pour aboutir à des récits» (B, 156). Les entrées inscrites dans les journaux d'enquête ont le caractère d'une étude génétique sur les textes écrits par les jeunes filles. Puisqu'un vrai journal n'a pas d'avant-texte, Lejeune déplace un peu le sens du mot et prouve que chaque note antérieure est le lieu d'apprentissages et d'évolutions pour les notes postérieures. Il suffit de comparer une note à une précédente pour voir «comment une écriture s'engendre elle-même par répétition ou par variation» $(\mathrm{B}, 317)$. Les études génétiques des journaux consisteraient donc à prendre pour point de départ le manuscrit original (la seule réalité du texte) et le considérer comme une destruction constante de ce qui se faisait tout au long de la notation. Même si c'est un peu une étude génétique à l'envers, elle est possible grâce à l'auto-commentaire qui est le trait pertinent de l'écriture diaristique. En relatant les étapes de son travail, il retrace l'histoire de la naissance des textes et du genre.

Comme tout diariste, Lejeune s'interroge sur la finalité du journal. Le texte abonde en réflexions métadiscursives qui contribuent à structurer la matière scientifique du livre. Le lecteur est obligé de procéder de la même façon que lorsqu'il lit n'importe quel journal intime, c'est-à-dire de suivre l'évolution de la pensée de l'auteur en regroupant incessamment le contenu des entrées. La théorie du journal s'élabore non seulement à partir de la lecture des manuscrits, mais aussi lorsque l'exploitation de l'expérience personnelle se fait dans le respect du protocole diaristique. Outre les informations en rapport avec l'enquète, le chercheur consigne beaucoup de renseignements sur sa manière de travailler et sur son activité d'universitaire. Bien des notes personnelles interviennent au moment du ralentissement momentané de l'enquête. Il discute souvent la question de l'authenticité de ses notes que certains spécialistes prennent pour un faux journal. Il remarque d'ailleurs que les personnes avec lesquelles il parle, s'intéressent davantage à son intérêt pour les journaux et non aux journaux eux-mêmes.

La méthode de travail qu'il a adoptée a aussi pour résultat l'élaboration d'une «théorie» de la recherche par le journal. Elle pourrait se résumer par les constatations suivantes: le journal lié à une circonstance précise a une fin, c'est-à-dire qu'une fois la recherche terminée, le journal est définitivement clos; le recours à ce genre de support permet au chercheur de garder dans un état relativement correct un rapport à soi et un rapport au temps; compte tenu de la discipline que nécessite l'approche 
scientifique du problème étudié, le journal évolue en tant que structure élaborée où l'examen de conscience du chercheur est plus sévère et plus systématique; le journal est le lieu de dialogue entre l'homme et le théoricien sur les questions méthodologiques; le caractère fragmentaire des entrées diaristiques favorise la fragmentation de bilans et de synthèses sur le vif. Malgré l'apparente paresse du chercheur et la rapidité du discours scientifique qui en résulte, la technique du journal de recherche témoigne selon lui de la transparence du travail scientifique et de l'ouverture du chercheur vis-à-vis de la question étudiée. L'expérience qui relève de la pratique de ce type de journal, facilite au chercheur l'approche des questions plus générales.

L'usage de l'une des formes d'expression diaristique pour des besoins de la recherche scientifique est incontestablement le prolongement de l'acte autobiographique. Dans un article sur la sincérité, publié dans «Autrement», Lejeune retrace d'une certaine manière les étapes de son travail universitaire et surtout les moments importants, voire les tournants de sa vie (les années 1956, 1969, 1986). Au fond ces étapes qui se succèdent et qui sont marquées par l'exercice de divers genres autobiographiques ne sont qu'un voyage aux pays du soi (cf. PA, 39) où tous les textes écrits racontent sa propre histoire. En publiant les livres-journaux sur les journaux, Ph. Lejeune ne fait qu'amorcer ce dont il rêve depuis qu'il a lu Le Temps immobile de Claude Mauriac, c'est-à-dire le montage de différents journaux.

Dans le journal d'automne 1992 qui clôt l'étude des journaux de jeunes filles, il a inséré une note tirée de son journal intime du 13 avril 1965 - «Je mens dès que j'ai une plume à la main. (...) Ce que j'écris dans mon journal est toujours plus ou moins faux, ou faussé. Je compose de moi une image truquée qui montre plus l'idée que je me fais de moi que ce que je suis vraiment. Mon romanesque personnel, ma sensibilité, tendent à me déformer à mes yeux à mon insu. - Souvent même, à moitié volontairement, je déforme la réalité, je brode, j'invente, pour réaliser un rêve. Et si je ne suis pas convaincu sur le moment, le temps qui efface en moi le souvenir donne de plus en plus de vérité à cette fiction» (MD, 431). Est-ce pour mentir moins qu'il a décidé de tenir le journal de recherche ou bien est-il en train, comme il sied à tous les diaristes, de réinventer le genre? En tout cas, le dispositif critique dont il se sert se veut comme un moyen de formation. D'une part c'est un récit sur les enjeux de l'évolution intellectuelle de l'homme et d'autre part c'est un traité théorique sur le genre même.

\section{JOURNAL INTIME D’ÉLÈVE EN DIFFICULTÉ}

Dans le cas de $\mathrm{Ph}$. Lejeune la réponse concernant la réinvention du genre pourrait être positive, car il appartient aux professionnels de l'écriture et il est en même temps praticien chevronné de l'art de l'autobiographie. En est-il de même pour les adeptes issus des ateliers d'écriture dont le succès, notamment aux États-Unis et en France, ne cesse de grandir? Sont-ils réellement en train de réinventer le genre, alors que tout 
leur fait défaut sauf l'envie d'apprendre à écrire des textes, y compris des textes autobiographiques'? Si l'on admet que tout s'apprend, la réponse est également positive.

Il est intéressant de savoir que l'enseignement en France initie, certes un peu inconsciemment, à l'écriture autobiographique au niveau de l'école primaire et au premier cycle du second (l'apprentissage de la rédaction, où l'emploi du «je» est sollicité). D'un effet inverse, l'apprentissage de la dissertation au second cycle a de quoi décourager les futures amateurs de l'écriture de soi. La situation est un peu paradoxale, car, exclue de l'enseignement, la pratique de l'autobiographie chez les adolescents témoigne d'une attitude réfractaire à la tradition de l'enseignement (PA, 24). Cependant les deux dernières décennies ont apporté des changements radicaux dans ce domaine. L'ouverture des ateliers d'écriture auprès des institutions scolaires, maisons de la culture ou centres des loisirs va au-devant du besoin irréssistible de l'expression personnelle. Ce n'est pas une invention française. Dans les pays anglophones l'apprentissage de l'autobiographie à l'aide des manuels, livres sur les thérapies par le journal ou guides de type «comment écrire son autobiographie» se fait depuis la fin des années soixante-dix, et il a été vite adopté par les Français ${ }^{3}$. Il est évident que les procédures appliquées dans ces manuels pratiques renvoient souvent à l'apprentissage des auto-bio-copies (qui est la prise en charge d'une intertextualité) et qu'elles proposent dans bien des cas des modèles de récits à contraintes $(\mathrm{B}, 14,139)$. L'écriture est donc faite de réemplois et son horizon d'attente est restreint à une illusion d'efficacité et d'originalité.

L'exemple que j'ai l'intention d'analyser est tiré non d'un manuel standard d'écriture autobiographique, mais d'un livre curieux édité par l'Éducation Nationale française en 1995. Il s'agit exactement du Cours d'Été du CNED (Centre National d'Enseignement à Distance), destiné aux élèves en difficulté. Trois catégories d'adolescents sont visées par le cours en question - ceux qui ont des résultats médiocres dans la matière, ceux qui ont eu une classe de seconde perturbée pour diverses raisons et ceux qui veulent reprendre la scolarité interrompue depuis plusieurs années. L'objectif est bien entendu de leur faciliter l'entrée en première. Le plan du cours comprend quatre séquences consacrées respectivement à quatre compétences dont l'essai de journal personnel ${ }^{4}$. Nous avons donc affaire à un drôle d'atelier d'écriture qui doit joindre l'utile à l'agréable.

Dès la première page de l'introduction l'élève est obligé de faire l'examen de conscience - mauvais souvenirs de classe, mauvaises notes, sentiment de nullité, suivi de conseils du professeur-ami qui lui fera aimer la lecture et l'écriture pendant les vacances. C'est vraiment une occasion unique d'apprendre à nager. Allez demander au stagiaire en diarisme de respecter les prescriptions aussi contradictoires - d'un côté liberté totale d'expression (faire vagabonder son esprit, noter ce qui vient à l'es-

\footnotetext{
${ }^{3}$ On trouvera une liste de guides d'autobiographie en langue anglaise dans Philippe Lejeune, Les brouillons de soi, éd. Seuil, Paris 1998, p.122.

${ }^{4}$ Les trois autres séquences sont consacrées à l'apprentissage des compétences suivantes: commentaire composé, argumentation et essai littéraire.
} 
prit, laisser couler les mots, adopter une attitude philosophique) et de l'autre - apporter le maximum de soin à l'écriture, à la rédaction et à la présentation, et naturellement, planifier bien le travail. Et puis, publiez-vous, c'est-à-dire, choisissez les meilleurs fragments (quatre à sept entrées inscrites à des dates différentes), recopiez-les et une fois des fautes d'orthographe et de syntaxe corrigées, envoyez le tout à votre lecteur et correcteur, votre ami et confident à la fois. Il y a même des suggestions pour le démarrage efficace: mettez-vous à l'écoute de vous-même et commencez par des choses les plus concrètes. Suit une note exemplaire: «Je suis dans le jardin. J'ai mon cahier sur mes genoux. Grand beau temps. La chaleur m'engourdit un peu. Ce n'est pas désagréable. Je n'ai pas très envie d'écrire. Quelle idée de nous demander de rédiger quelques pages de journal!» (AN, 6). Certes, ce n'est pas une recette miracle, mais comme le déclare le professeur, partagez «le plaisir que j'éprouve moi-même à lire et à écrire» (AN, 4). Le plaisir de lire, le professseur l'aura bientôt. Il reste à savoir si l'élève aura le plaisir d'écrire, et d'abord celui de lire un cours intitulé «Plaisir d'écrire». À chacun ses plaisirs.

Avant de passer à la pratique commandée du journal, l'élève suit l'exposé sur le journal d'Anaïs Nin, précêdé d'un aperçu sur les origines historiques du journal intime et suivi de quelques témoignages de diaristes, recueillis dans "Cher cahier..." de $\mathrm{Ph}$. Lejeune. Nicole Jouen invite le retardataire à lire la séquence et à noter dans l'immédiat ses impressions. Comme il s'agit d'«un livre de lecture accompagnée par le commentaire et la présence d'un professeur», les éditeurs n'ont pas manqué de prévenir qu'il exige un effort d'attention et une totale disponibilité. C'est ce que j'ai essayé de faire en me prêtant au jeu.

L'historique du journal fait entrevoir en quoi consiste la pratique, quelle est la différence entre «journaliste» et «diariste» et comment devenir un peu Anne Frank, Maine de Biran, Maurice de Guérin, servis à la sauce Michel de Montaigne pour être sûr de réussir cette écriture singulière qui, comme on nous l'explique, ne concerne qu'un seul individu et qui apparaît comme quelque chose de bizarre, d'étrange, d'étranger. Bref, un individu singulier s'adonne à une activité singulière. Dès le départ l'élève sait qui il est et ce qu'on attend de lui. A partir d'informations fournies on peut conclure que l'adolescent tiendra en même temps le journal de déception qui lui servira à panser la blessure et à surmonter l'épreuve, et le journal d'exploration que pratique une personne submergée par trop d'événements qu'elle n'arrive pas à maîtriser. Le lycéen vit vraiment une situation dramatique. Le journal intime qu'il ouvre en lisant les propos du correcteur sera donc sa réponse à une crise qui le tracasse par l'intermédiaire des arguments suivants: y voir clair en soi-même, faire face à l'isolement, comprendre le sens de la vie, se recentrer et mieux maîtriser sa vie. Ouff! c'est vraiment compliqué de tenir le journal pendant les vacances!

Le deuxième volet de l'exposé concerne le journal d'Anaïs Nin (1903-1977, écrivain de langue anglaise). Le professeur fait le compte-rendu commenté des fragments choisis selon l'objectif précis qui est le suivant: avant que l'élève se lance dans la pratique du journal, il faut lui faire croire que son drame n'a rien d'exceptionnel et 
qu'une autre personne l'a déjà vécu au début du siècle. La lecture est basée sur deux volumes du journal d'enfance d'Anaïs qu'elle a commencé à l'âge de 11 ans, au moment du tournant important dans la vie de la jeune fille (la mère, abandonnée par son mari, célèbre pianiste, quitte l'Europe et s'installe avec les enfants aux États-Unis). Quand les arguments didactiques lui font défaut (le journal d'enfant n'est pourtant pas un traité de pédagogie), la commentatrice recourt au journal d'adulte d'Anaïs. Cette dẻmarche a de quoi rendre schizophrène la situation d'un adolescent, car on lui demande d'être deux en un, enfant et homme adulte, en l'occurence jeune fille et femme d'une quarantaine d'année.

Pour Anaïs le tournant de la vie est marqué par l'ouverture du cahier et l'inscription, sous forme d'un testament, du profond sentiment de nostalgie et de séparation. Elle inscrit le destinataire virtuel (mélange du journal personnifié et des proches indéterminés), et construit de nombreux portraits des membres de sa famille dont le père, illustrés par des photos, dessins ou poèmes. Affligée, et aussi pour fuir le regard jeté par-dessus son épaule par sa mère, la fille se retire de plus en plus dans son jardin secret où elle s'épanche en solitaire. Derrière les fragments habilement sélectionnés et commentés, résonne la voix astucieuse du professeur qui invite à suivre exactement les traces d'Anaïs. Pour si évidentes qu'elles soient, les manipulations d'un élève sont focalisées sur l'adoption par lui de la démarche d'Anaïs et sur l'acceptation du correcteur en tant que confident (la virtualité de celui-ci n'est plus qu'une singerie du procédé diaristique, car plus que toute autre personne, le professeur est identifiable). Lorsque Anaïs exprime la volonté de réécrire un passage de son journal, le correcteur en profite pour rejouer sur l'exigence littéraire des notes que l'élève donnera bientôt à lire.

Si l'on admet que le commentaire facilite la mise en pratique du devoir imposé par le professeur, il est pourtant difficile d'accepter qu'il dérive vers la pression psychique continue. Peut-être l'élève n'aurait-il rien contre la tenue du journal s'il pouvait parler librement des choses selon les modalités qui sont les siennes et en accord avec sa situation psychique du jour. Rêver un peu, noter vraiment ce qui vient à l'esprit, relater de petits faits divers, même si cela ne plaît pas à ce drôle d'ami virtuel. Hélas, la vigilance du professeur ne se laisse pas endormir. Il veille! Il pratique cette sorte de terrorisme psychique qui consiste à faire souffrir l'élève comme a souffert Anaïs Nin, à cette différence près que sa souffrance était spontanée et justifiée par les circonstances. Une réflexion de la diariste: «Aujourd'hui je n'ai rien à dire (...) en ouvrant un livre au hasard j'ai lu: «La vie n'est qu'une triste réalité»», laquelle dans les confidences d'Anaïs ouvre un long passage sur la blessure psychique et la souffrance physique, est servie ici comme exemple d'une expérience à vivre par le lycéen. La vivre et la partager avec un être bienveillant et muet (le professeur sans doute; d'ailleurs à aucun moment du cours on n'envisage de coopérer avec les parents d'élève; ils ne sont même pas mentionnés par le professeur). Au lieu de faire souffrir les proches, l'élève n'a qu'à faire souffrir son journal, comme Anaïs. 
La formule du cours à distance ne permet pas de connaître la situation réelle de l'adolescent en question et encore moins la cause de son échec scolaire. Les difficultés qu'il vit ne relèvent pas forcément des expériences traumatisantes de son passé comme voudrait le faire croire le correcteur. Cependant selon sa vision des choses, le journal n'est possible que si l'élève réussit à retrouver des expériences qui auraient pu le marquer quand il était petit. Ya-t-il des moyens plus efficaces pour culpabiliser l'adolescent qui n'attachait peut-être jamais d'importance à tel ou autre incident de son passé? Les modalités proposées par le correcteur ont pour but de forcer une écriture aussi sincère et non censurée qu'artificielle et adiaristique. L'écriture sur commande, du moins dans ce cas, ne fera donc jamais du carnet un ami muet et compatissant comme c'était le cas d'Anaïs. Pour le journal d'adolescent le rôle de juge et de témoin de ses progrès est attribué d'office au professeur qui en lira certaines pages. Puisque le but principal de ce devoir de vacances est de mobiliser l'élève à l'apprentissage de l'écriture, le correcteur joue sur le double mouvement de la notation diaire (préserver l'intimité et la faire partager aux autres) afin de justifier son voyeurisme. Bien que la contradiction entre préservation et divulgation est typique de tous les journaux intimes écrits de manière spontanée, la question même paraît embarrassante si elle fait partie du projet d'écriture. Or, le professeur est parfaitement conscient que le débutant en diarisme accepterait difficilement une telle notion d'intimité. Pour le rassurer, mais en même temps pour atténuer l'effet de l'écriture commandée, il étale devant son élève des fragments extraits de différents journaux d'Anaïs qui illustrent des étapes successives de son évolution de diariste et de créateur. Au fond on demande à l'élève de conserver la mentalité d'enfant et la technique d'adulte.

Le choix du journal d'Anaïs Nin à des fins didactiques s'explique aussi par sa valeur thérapeutique. Le journal sert à la jeune fille d'exutoire par où elle se débarrasse de mauvais souvenirs et expériences. Tout en se relisant, elle est en train de prolonger ses rêveries et de s'inventer. Dans cette perspective l'écriture devient pour Anaïs un acte de purification et d'auto-création, et bien entendu un moyen d'envisager un avenir radieux et confiant. L'attitude d'Anaïs n'a rien d'original et elle est valable pour beaucoup de diaristes. Ces stéréotypes facilitent la tâche du professeur, car il est dispensé de moraliser en son nom ce qui aurait fini par décourager l'élève. Et pourtant le commentaire est loin d'être exempt de ton moralisateur. Le professeur a sélectionné les passages selon une double formule du personnage interposé. D’une part, la jeune Anaïs s'adresse directement à un compagnon du même âge pour lui montrer que la tenue du journal peut remédier à ses difficultés. L'élève se met donc à l'écoute de l'âme-soeur qui débroussaille la première le chemin. D'autre part Anaïs adulte, qui dispose déjà d'un savoir-faire nécessaire, raconte au lecteur ce qu'il faut faire pour réussir et comment elle-même elle a réussi grâce à l'écriture. En laissant parler la femme-écrivain, le professeur évite ainsi d'ennuyer l'élève et lui fait partager l'optimisme de quelqu'un qui s'est remis pour de bon des difficultés éprouvées à l'âge d'adolescence. Le tripotage dans divers journaux de Nin aurait de quoi inquiéter 
le spécialiste de journaux intimes, hostile au mélange de registres d'enfant et d'adulte. Effectué pourtant à l'usage d'un retardataire, il peut être bénéfique: l'élève sait d'avance qu'il va réussir.

L'édification de l'élève, voire sa réussite scolaire, ne saurait être satisfaisante uniquement par le biais du relèvement moral d'Anaïs Nin. Le repèchage demande bien entendu des moyens concrets. Le professeur ne manque pas d'en proposer quelques-uns en puisant toujours dans le contenu du journal. Il revient sur les méthodes de rédaction du texte et explique comment Anaïs construisait des portraits, de quelle manière elle rédigeait ses notes ou bien à quel vocabulaire elle faisait appel. Les questions de style sont longuement analysées à partir d'autoportraits ou lettres fictives de la jeune fille. L'exemplification est très détaillée et son but est d'éveiller chez l'élève le goût de la lecture, condition sine qua non de savoir bien écrire.

La manipulation à visée didactique est la plus perceptible lorsque le commentaire aboutit aux premiers et sérieux bilans qu'Anaïs fait à l'âge de 16-17 ans (c'est bien l'âge du lycéen). Le professeur la laisse parler pour témoigner de sa victoire par le journal. Ainsi lisons-nous entre autres que le journal lui a permis de se réconcilier avec la vie, qu'il trace l'itinéraire d'une renaissance et qu'il est devenu un acte de foi dans la vie. Privées de contexte, les informations sur les qualités de l'écriture diaire deviennent simplistes et perdent bien sûr de leur authenticité. Banalisées à l'extrême, elles s'imposent à l'adolescent comme un modèle d'une pratique controuvée et difficile à réaliser dans le cadre d'un cours de rattrapage. L'élève accepterait-il facilement un tel défi? Je ne pense pas à moins qu'il n'apporte à cette occasion sa codicille à un certain renouvellement de la forme du journal. Compte tenu de son échec scolaire, c'est peu probable, mais tout de même c'est possible s'il arrive à maîtriser l'art du montage et à proposer un texte convaincant à partir d'une écriture particulière.

Pour si différents qu'ils soient, les deux dispositifs que j’ai présentés ont malgré tout plusieurs traits communs. Tout d'abord ils se veulent comme des moyens de formation. D'une part il est question de l'élaboration de la théorie du journal qui est en train de se faire lors de la notation et qui devient en même temps un récit sur les enjeux de la formation intellectuelle de l'individu. D'autre part, le journal est choisi comme un outil de formation scolaire dont le but est avant tout d'aider un élève à sortir d'une mauvaise passe et de lui faire retrouver le goût de lire et d'écrire. De manière un peu paradoxale, ce type d'atelier d'écriture rejoint les pratiques de scolarisation par le journal qui se sont développées au XIX ${ }^{\mathrm{e}}$ siècle et dont Philippe Lejeune a relaté l'histoire dans ses journaux de recherche. Un autre trait commun se rapporte aux contraintes dorit sont marquées ces deux dispositifs. Lejeune tient à décharger ses entrées d'inscriptions trop personnelles et à s'imposer des règles du discours scientifique qui serait à la fois autobiographique. Le journal à contraintes proposé à l'adolescent est la mise en charge de l'intimité à l'usage d'un exercice de lecture et d'écriture. Les deux cas analysés ne sont certainement pas représentatifs de l'histoire du journal, mais ils témoignent à leur manière de l'évolution constante du genre et de sa codification en cours. 


\section{BIBLIOGRAPHIE}

(CC) - «Cher cahier...». Témoignages sur le journal personnel, recueillis et présentés par Philippe Lejeune, éd. Gallimard, Paris.

(MD) - P. Lejeune, Le moi des demoiselles. Enquête sur le journal de jeune fille, éd. Seuil, Paris 1993.

(WSP) - Ph. Lejeune, L'autobiographie ordinaire, dans: Regina Lubas-Bartoszyńska, Michel Braud (dir.), Où en sommes-nous dans les recherches autobiographiques?, Prace Romanistyczne 4, Wydawnictwo Naukowe WSP, Kraków 1994, pp. 7-15.

(L) - L. Desmoulins, Journal 1788-1793, texte établi et présenté par Pihlippe Lejeune, éd. Editions des Cendres, Paris 1995.

(TT) - Le tournant d'ıme vie, Philippe Lejeune, Claude Leroy (dir.), éd. RITM, Université Paris $\mathrm{X}$, Paris 1995.

(S) - Ph. Lejeune, Sincérité, «Autrement» 1995; repris dans: Philippe Lejeune, Pour l'autobiographie. Chroniques. Cf. (PA).

(AN) - N. Jou en, Français. Objectif Première. Cours d'Été du CNED, éd. Education Nationale, Paris 1995.

(AP) - L'autobiographie en procès, Philippe Lejeune (dir.), éd. RITM, Universitć Paris X, Paris 1997.

(TJ) - Ph. Leje une, Tenir un journal. Histoire d'une enquête (1987-1997), «Poétique», N 111 , septembre 1997, pp. 359-381.

(PA) - Ph. Lejeune, Pour l'autobiographie. Chroniques, éd. Seuil, Paris 1998.

(B) - Ph. Lejeune, Les brouillons de soi, éd. Seuil, Paris 1998. 